\title{
Circulating miRNA levels differ with respect to carotid plaque characteristics and symptom occurrence in patients with carotid artery stenosis and provide information on future cardiovascular events
}

\author{
Rafal Badacz' ${ }^{1}$ Tadeusz Przewłocki ${ }^{1}$, Jacek Gacoń², Ewa Stępieńn ${ }^{3}$, Francisco J. Enguita ${ }^{4}$, Izabela Karch ${ }^{1}$, \\ Krzysztof Żmudka ${ }^{1}$, Anna Kabłak-Ziembicka ${ }^{1}$

\begin{abstract}
${ }^{1}$ Department of Interventional Cardiology, School of Medicine, Jagiellonian University, John Paul II Hospital, Krakow, Poland ${ }^{2}$ Department of Invasive Cardiology, E. Szczeklik's Hospital, Tarnow, Poland

${ }^{3}$ Department of Medical Physics, Marian Smoluchowski Institute of Physics, Faculty of Physics, Astronomy and Applied Computer Science, Jagiellonian University, Krakow, Poland

${ }^{4}$ Institute of Molecular Medicine, Faculty of Medicine of Lisbon, Lisbon, Portugal
\end{abstract}

Adv Interv Cardiol 2018; 14, 1 (51): 75-84 DOI: https://doi.org/10.5114/aic.2018.74358

\begin{abstract}
A bstract
Introduction: Circulating microRNAs (miRNAs) levels are potentially important biomarkers and therapeutic targets of cerebral ischemic event (CIE) in patients with internal carotid artery stenosis (ICAS).

Aim: This prospective study investigated associations between circulating miRNAs and symptomatic and asymptomatic ICAS, carotid plaque morphology and future cardiovascular events.

Material and methods: Circulating miRNAs (miR-1-3p, miR-16-5p, miR-34a-5p, miR-124-3p, miR-133a-3p, miR-133b, miR-134-5p, miR-208b-3p, miR-375 and miR-499-5p) were analyzed in 92 consecutive patients with significant ICAS referred for revascularization. Group I comprised 65 subjects (41 males, age $69.3 \pm 9.7$ years) with a recent CIE. Group II comprised 27 patients (15 males, age $68.2 \pm 8.4$ years) with asymptomatic ICAS. The ICAS degree and plaque morphology was assessed by ultrasonography. The incidences of cardiovascular death (CVD), myocardial infarction (MI) and recurrent CIE (CVD/MI/CIE) were recorded prospectively (mean: $38.7 \pm 3.8$ months).

Results: Groups II and I differed significantly in levels of miR-124-3p ( $p=0.036)$, miR-133a-3p $(p=0.043)$ and miR-134-5p $(p=0.02)$. Hypoechogenic, as compared to echogenic, plaques differed in levels of miR-124-3p $(p=0.038)$, miR-34a-5p $(p=$ $0.006)$, miR-133b ( $p=0.048)$, miR-134-5p ( $p=0.045)$, and miR-375 $(p=0.016)$, while calcified plaques differed in miR-16-5p $(p=0.023)$. Ulcerated plaques showed higher levels of miR-1-3p $(p=0.04)$ and miR-16-5p $(p=0.003)$, while thrombotic plaques showed lower levels of miR-1-3p $(p=0.032)$. CVD/MI/CIE occurred in $14(15.5 \%)$ out of 90 follow-up patients. Multivariate Cox and ROC analysis showed associations between miR-1-3p and CVD (AUC = 0.634; $\mathrm{HR}=4.84 ; 95 \% \mathrm{Cl}$ : 1.62-14.5; $p=0.005)$, MI (AUC = 0.743; HR = 7.8; 95\% Cl: 2.01-30.0; $p=0.003)$, and CVD/MI/CIE (AUC = 0.560; $\mathrm{HR}=4.6 ; 95 \% \mathrm{Cl}: 1.61-13.1$; $p=0.004)$, while miR-133b was associated with recurrent $\mathrm{CIE}(\mathrm{AUC}=0.581 ; \mathrm{HR}=2.25 ; 95 \% \mathrm{Cl}: 1.01-5.02 ; p=0.047)$.

Conclusions: A significant difference in levels of selected miRNAs is observed in symptomatic vs. asymptomatic ICAS. Plaque morphology and structure is associated with change of miRNA levels. The expression of miR-1-3p may be a potential prognostic factor for future cardiovascular events.
\end{abstract}

Key words: ischemic stroke, internal carotid artery stenosis, miRNAs, biomarkers, carotid plaque morphology, prognosis.

\section{Introduction}

In the developed countries, cerebral ischemic events (CIE) have a major impact on public health as the first cause of long-term disability and the fifth leading cause of death [1]. Each year approximately 795 million people suffer from $\mathrm{CIE}$ worldwide, with mortality rates ranging from $16.7 \%$ to $38.6 \%$ for recurrent IS [2]. Cerebral ischemic events survivors remain at significant risk of recurrent neurological and cardiac ischemic events [1, 3].

Corresponding author:

Prof. UJ Anna Kabłak-Ziembicka MD, PhD, Department of Interventional Cardiology, School of Medicine, Jagiellonian University, John Paul II Hospital, 80 Prądnicka St, 31-202 Krakow, Poland, phone: +48 1261435 01, e-mail: kablakziembicka@op.pl Received: 31.10.2017, accepted: 29.12.2017. 
In the NDPSS study, the cumulative 2-year stroke recurrence rate, irrespective of etiology, was $10.8 \%$, while the Scandinavian study showed that large artery disease was related to $19.2 \%$ stroke recurrence at 2 years, as compared to $4.9 \%$ for lacunar stroke, $8.2 \%$ for cardioembolic and $5.6 \%$ for cryptogenic, and $12.8 \%$ in patients with an undetermined CIE cause [2].

Internal carotid artery stenosis (ICAS) accounts for about $20 \%$ of all cases of CIE. The etiology of CIE associated with ICAS may be cerebral hypoperfusion due to high-grade ICAS, or more frequently emboli release from an unstable carotid plaque irrespective of stenosis degree [3-7]. Several studies have demonstrated that carotid plaque morphology should be taken into consideration when assessing the risk of $\mathrm{CIE}$, and timing for carotid artery revascularization $[8,9]$.

Echolucency and heterogeneous appearance in the ultrasonography, as well as the presence of thrombus or ulcerations within a plaque, are distinguishing features for an unstable plaque with a predisposition to rupture [8-10]; however, ultrasonography alone is insufficient to diagnose the plaque type accurately in some patients [11, 12].

Thus, the search for more relevant biomarkers is justified to indicate high-risk subsets of patients with ICAS. Furthermore, the ischemic event in one arterial territory predisposes to vascular events in the other territory $[13,14]$.

Thus, further research on new biomarkers to expedite diagnosis and long-term prognosis of $\mathrm{CIE}$ seems reasonable. One promising area is evaluation of the change in circulating microRNAs following CIE in both animal and human models [15-17]. Furthermore, circulating miRNAs appear to remain stable in exosomes present in blood serum, even in extreme conditions such as high temperature or changing $\mathrm{pH}$ and in spite of existing RNases, which makes them the perfect candidates for biomarkers in a complex pathological process such as CIE $[18,19]$. Although the above-mentioned extreme conditions are not characteristic for in vivo studies, the sampling must be managed promptly and without delay in clinical circumstances.

Accumulating experimental and clinical evidence improved our knowledge about the role of miRNAs in cellular and molecular processes affecting atherosclerotic plaque initiation and progression [20, 21]. Nevertheless, the role of miRNA-regulated processes in the echogenicity and surface composition of atherosclerotic plaque is still not revealed.

\section{Aim}

The present study aimed to compare expression of serum miRNAs in patients with recent CIE and asymptomatic patients with severe ICAS. We tried to evaluate the correlation of miRNA concentration and stenosis degree as well as to determine miRNA levels with respect to carotid plaque morphology assessed by means of ultrasonography. We also assessed the predictive value of selected specific miRNAs in long-term cardiovascular prognosis.

\section{Material and methods}

\section{Patient selection}

This prospective study included 92 consecutive patients (56 men; mean age: $69 \pm 9.3$ years) with significant ICAS (mean stenosis degree: $79.4 \pm 12 \%$ ), referred to the Department of Vascular and Endovascular Surgery for carotid artery revascularization.

Group I comprised 65 subjects (41 men aged 69.3 \pm 9.7 years) who suffered from CIE within 12 weeks (median time 26 days) before admission. Group II comprised 27 patients (15 men aged $68.2 \pm 8.4$ years) with asymptomatic ICAS.

Inclusion criteria were as follows: age over 18 years, presence of significant ICAS referred to carotid artery revascularization.

Exclusion criteria included: occluded ICA, atrial fibrillation or other known potential causes of CIE (coagulation disorders, etc.), coronary instability, congestive heart failure in class III or IV, chronic or acute inflammatory status, active cancer, evidence of recent intracranial bleeding.

All subjects signed their informed consent prior to carotid intervention in accordance with the requirements of the institutional local Ethics Committee. The study was performed consistently with the requirements of the Declaration of Helsinki.

\section{Neurological assessment}

The data of the index CIE (ischemic stroke or transient ischemic attack (TIA)) were obtained from a stroke unit, and sourced from available medical documentation, brain imaging either with computed tomography (CT) or magnetic resonance imaging (MRI).

Additional neurological assessment was performed in all subjects to confirm the indications for carotid revascularization.

\section{Carotid ultrasound}

Every patient had high-resolution B-Mode, color Doppler and pulse Doppler ultrasonography of extracranial arteries performed on admission to the hospital with the ultrasound machine Toshiba Aplio Power Vision (Toshiba Medical Systems Co, Ltd, Tokyo, Japan) equipped with 4-11 MHz linear array transducer). Patients were examined in a supine position with the head tilted backwards.

In compliance with the Carpenter criteria, significant ICAS was assessed through measuring an increase in the peak systolic velocity $(P S V)>2.1 \mathrm{~m} / \mathrm{s}$ and the end-diastolic velocity $(E D V)>0.7 \mathrm{~m} / \mathrm{s}$ [22]. The degree of ICAS was also assessed according to NASCET criteria [23], by measuring the vessel diameter at the point of maximal stenosis compared with the plaque-free vessel diameter distally from the lesion.

The plaque morphology was analyzed by the modified criteria [24, 25]. The lesions were assessed by their 
echogenicity and were divided into: echolucent, e.g. soft, lipid rich; moderately echogenic, e.g. heterogeneous; or fibrotic and dominantly echogenic, e.g. calcified [24]. The surface structure was examined for the presence of ulcerations or thrombus, and defined as ulcerative or smooth and thrombotic, respectively [25].

\section{Angiography and ICAS revascularization}

Patients were selected for CAR if they had symptomatic $>50 \%$ or asymptomatic $>80 \%$ lumen stenosis evaluated by ultrasonography and/or computed tomography or quantitative angiography.

The choice of revascularization method, carotid artery stenting (CAS) or carotid endarterectomy (CEA), was made by the multidisciplinary working team including a neurologist, vascular surgeon, endovascular specialist, cardiologist, and radiologist. On patient referral, clinical presentation, accompanying comorbidities (e.g. renal dysfunction, respiratory tract disease, access site, coronary artery disease), anatomic assessment (limited surgical access, prior cervical irradiation, prior ipsilateral CEA, and contralateral carotid occlusion), data from imaging workup, as well as method feasibility and safety were taken into consideration.

The detailed CAS technique was described previously [25]. In brief, the technique was at the operator's discretion, provided that the 'tailored-CAS' algorithm was applied, allowing for optimal choice of neuroprotection device and stent type, depending on lesion morphology and the neurological status of the patient [26].

The CEA technique was at the operator's discretion, with preference of eversion technique with periprocedural shunt whenever feasible [27].

Periprocedural medical management included antiplatelet treatment. All patients obtained optimal medical treatment in concordance with recommendations of relevant societies $[23,28]$.

\section{MicroRNA extraction and profiling}

All blood samples were collected on patient admission to the department, prior to any intervention, immediately after the signed informed consent was obtained from the patients.

Samples were allowed to coagulate for $30 \mathrm{~min}$, centrifuged and sera were frozen at $-80^{\circ} \mathrm{C}$ until miRNA and selected biomarkers' analysis. Extraction of miRNA was performed by means of the miRNeasy Serum/Plasma Kit (cat. No. 217184, Qiagen) with the beginning lysis by Trizol LS Reagent (cat. No. 10296-028, Invitrogen). The RNA yield and concentrations were determined by capillary electrophoresis on the Agilent Bioanalyser 2100 with the Eukaryote Total RNA Pico Chip (Agilent Technologies, Inc, Santa Clara CA). An average $60 \pm 31.9 \mathrm{pg} / \mu \mathrm{l}$ of total RNA from $300 \mu$ l of serum was recovered.
Circulating miRNAs (muscle: miR-1-3p, miR-133a-3p, miR-133b, miR-208b-3p and miR-499-5p; brain: miR34a-5p, miR-124-3p and miR-134-5p; liver miR-122-5p; and pancreas miR-375) in peripheral blood from serum samples were analyzed in each case.

Our choice for selection of particular miRNAs was based on database research (PubMed). Analyzed miRNAs were taken into consideration based on the data regarding their potential relationship with development of atherosclerosis (usually based on experience in patients with coronary and peripheral artery disease) and their potential prognostic value. Additionally, we included miRNAs which were discriminating biomarkers for coronary artery vessel occlusion in patients with acute coronary syndrome [29].

At the time of the study, Exiqon LNA primers were used to quantify 10 mature miRNAs using the ViiA 7 real-time PCR system equipped with a 384-well reaction plate (Life Technologies). RNA was converted to cDNA using the Universal cDNA Synthesis Kit (cat. No. EQ-203300, Exiqon). Before synthesis, RNAs were spiked with a synthetic miRNA that served as a control the cDNA synthesis reaction. Real-time PCR was performed in triplicate with SYBR Green master mix Universal RT (cat. No. EQ-203400, Exiqon) using standard conditions. The organ-specific candidate miRNAs were selected: cardiac and skeletal muscle (miR-1-3p, -133a-3p, -133b, -208b-3p, -499-5p), brain-related (miR-34a-5p, -124-3p, -134-5p), liver (miR122-5p), pancreas (miR-375) and a reference miRNA for serum (miR-16-5p). The fold changes (RQ) were calculated. Hierarchical clustering of median-normalized miRNA values was performed using Cluster 3.0 software [15].

\section{Follow-up}

The incidence of cardiovascular death (CVD), myocardial infarction (MI) and recurrent $\mathrm{CIE}$, as well as composite end-point (CVD/MI/CIE), was recorded prospectively during the mean follow-up period of $38.7 \pm 3.8$ months (range: 3-50 months).

Myocardial infarction was diagnosed according to current criteria of the European Society of Cardiology [27]. Diagnosis of CIE was confirmed by a neurologist to ensure reliability. CVD was defined as a fatal CIE, fatal $\mathrm{MI}$, or other cardiovascular death (i.e. any sudden or unexpected death unless proven as non-cardiovascular on autopsy).

\section{Statistical analysis}

Continuous variables are presented as mean \pm SD, while categorical variables are expressed as frequencies and percentages. Means of analyzed parameters across groups were tested with the analysis of variance (ANOVA) test, and frequencies were compared by the $\chi^{2}$ test for independence. 
The normal distribution of studied variables was determined by the Shapiro-Wilk test. Differences between mean values of miRNAs were verified using the Mann-Whitney $U$ test as the distribution of variables was found not to be normal.

The potential independent prognostic markers of cardiovascular events during the follow-up period were established from the clinical, procedural, and angiographic variables with a univariate Cox proportional hazard analysis, and if $p<0.1$ they were entered into a multivariate Cox proportional hazard model. The results of the multivariate Cox analysis were expressed as relative risk (RR) and $95 \%$ confidence interval $(95 \% \mathrm{Cl})$.

The receiver operating characteristic curves (ROC) were calculated for independent miRNAs predictive of cardiovascular event with assessment of the area under the ROC curve (AUC), 95\% Cl as well as sensitivity and specificity of the cut-off miRNA value that best discriminates risk of CVD, $\mathrm{MI}$ and IS.

Statistical analyses were performed with Statistica 12.0 software. Statistical significance was assumed at $p<0.05$.

\section{Results}

Baseline characteristics of study participants are shown in Table I. The groups did not differ significantly in any of the analyzed parameters.
In comparison to group II, group I showed significant differences in miR-124-3p (0.58 \pm 1.68 vs. $0.38 \pm 0.67$; $p=0.036)$, miR-133a-3p (1.18 \pm 2.53 vs. $1.48 \pm 1.99$; $p=0.043)$ and miR-134-5p (7.74 \pm 41.96 vs. $1.77 \pm 4.4$; $p=0.02$ ) levels, while there was no difference in levels of the other investigated miRNAs (Figure 1). Thus, the significant differences were observed in brain derived miRNAs exclusively.

There were no significant differences with regard to plaque surface and carotid stenosis degree between group I and group II (Table II). There was no correlation between degree of ICAS either on ultrasonography or on angiography and miRNA levels.

In symptomatic patients, echolucent and moderately echogenic plaques were more prevalent than hyperechogenic plaques, as compared to asymptomatic subjects $(p=0.033)$ (Table II).

In comparison to soft and thrombotic echolucent plaques, the moderately echogenic plaques showed significantly higher levels of miR-124-3p (1.159 \pm 2.9 vs. $0.33 \pm 0.4 ; p=0.038)$, miR-134-5p (19.5 \pm 73.3 vs. 1.98 $\pm 4.39 ; p=0.045)$, miR-34a-5p (2.03 \pm 7.23 vs. $1.11 \pm 1.41$; $p=0.006)$ and miR-375 (81.1 \pm 156.3 vs. $16.63 \pm 36.7 ; p=$ $0.027)$, and lower levels of miR-133b (1.74 \pm 1.49 vs. 2.62 $\pm 4.28 ; p=0.048$ ) (Figure 2). Non-significant differences were noted for miR-16-5p (353.31 \pm 831.3 vs. 408.32

Table I. Patients characteristics

\begin{tabular}{|c|c|c|c|}
\hline Parameter & $\begin{array}{l}\text { Symptomatic patients } \\
\qquad(n=65)\end{array}$ & $\begin{array}{l}\text { Asymptomatic patients } \\
(n=27)\end{array}$ & $P$-value \\
\hline Age, mean \pm SD [years] & $69.3 \pm 9.7$ & $68.2 \pm 8.4$ & 0.415 \\
\hline Male, $n(\%)$ & $41(63.1)$ & $15(55.6)$ & 0.502 \\
\hline Smoking, $n(\%)$ & $39(60)$ & $15(55.6)$ & 0.693 \\
\hline $\mathrm{CAD}, n(\%)$ & $31(47.7)$ & $17(63.0)$ & 0.181 \\
\hline Previous MI, $n(\%)$ & $12(18.5)$ & $8(29.6)$ & 0.236 \\
\hline Previous CABG, $n(\%)$ & $3(4.6)$ & $4(14.8)$ & 0.092 \\
\hline Previous PCI, $n$ (\%) & $6(9.2)$ & $5(18.5)$ & 0.211 \\
\hline PAOD, $n(\%)$ & $16(24.6)$ & $9(33.3)$ & 0.392 \\
\hline Hypertension, $n$ (\%) & $61(93.8)$ & $27(100)$ & 0.187 \\
\hline Diabetes, $n(\%)$ & $25(38.5)$ & $9(33.3)$ & 0.642 \\
\hline Dyslipidemia, $n$ (\%) & $54(83.1)$ & $24(88.9)$ & 0.691 \\
\hline Renal dysfunction, $n$ (\%) & $15(23.1)$ & $6(22.2)$ & 0.929 \\
\hline Fibrinogen, mean $\pm \mathrm{SD}[\mathrm{g} / \mathrm{l}]$ & $3.91 \pm 1.27$ & $3.50 \pm 1.03$ & 0.148 \\
\hline Hs-CRP, mean \pm SD [g/l] & $4.39 \pm 5.72$ & $3.68 \pm 3.34$ & 0.783 \\
\hline LDL-C, mean \pm SD $[\mathrm{mmol} / \mathrm{l}]$ & $2.74 \pm 1.00$ & $2.56 \pm 0.99$ & 0.311 \\
\hline Creatinine, mean $\pm S D[\mu \mathrm{mol} / \mathrm{l}]$ & $89.9 \pm 33.66$ & $87.1 \pm 22.2$ & 0.597 \\
\hline
\end{tabular}

CABG - coronary artery bypass grafting, CAD - coronary artery disease, LDL-C - low-density lipoprotein cholesterol, LVEF - left ventricular ejection fraction, $M I-$ myocardial infarction, $P A O D$ - peripheral artery occlusive disease. 
$\pm 804.8 ; p=0.077)$, miR-208b-3p $(0.7 \pm 2.97$ vs. $0.27 \pm 1.9$; $p=0.051)$ and miR-1-3p ( $p=0.085)$ (Figure $2 \mathrm{~A})$.

In comparison to the moderately echogenic plaques, the calcified hyperechogenic plaques showed significantly lower levels of miR-34a-5p (1.1 \pm 1.39 vs. $2.0 \pm 7.2 ; p=$ $0.017)$, miR-134-5p ( $2.4 \pm 5.29$ vs. $19.5 \pm 73.3 ; p=0.042)$, and miR-375 (21.5 \pm 43.9 vs. $81.1 \pm 156.3 ; p=0.046)$, and higher levels of miR-16-5p (504.1 \pm 941.8 vs. 353.3 $\pm 831.3 ; p=0.029$ ) (Figure $2 \mathrm{~B}$ ).

Furthermore, in comparison to hypoechogenic plaques, the calcified hyperechogenic plaques showed significantly higher levels of miR-16-5p (493.6 \pm 934 vs. $211.9 \pm 371 ; p=0.023$ ) (Figure $2 \mathrm{C}$ ).

Ulcerated plaques were characterized by higher levels of miR-1-3p (0.36 \pm 0.39 vs. $0.20 \pm 0.21 ; p=0.049)$ and miR-16-5p (652 \pm 1130 vs. $283 \pm 591 ; p=0.003)$ (Figure $2 D$ ), was related to thrombus formation with lower levels of miR-1-3p (0.11 \pm 0.09 vs. $0.26 \pm 0.29 ; p=0.032$ ), as compared to non-ulcerated and non-thrombotic plaques, respectively.

Eventually, 75 patients were referred for CAS, 17 for CEA. Of those, CAR was performed in 83 subjects, while 9 patients were eventually treated conservatively. Thirty-day periprocedural complications occurred in 4 (4.8\%) subjects, including 2 deaths as a consequence of hyperperfusion syndrome with subsequent intracranial hemorrhage ( 1 after CAS and 1 after CEA), 1 TIA and 1 minor ischemic stroke. No MIs were observed in the perioperative period.

During the mean follow-up period of $38.7 \pm 3.8$ months (range: 3-50 months), out of 90 subjects, CVD/ $\mathrm{MI} / \mathrm{CIE}$ occurred in 14 (15.6\%) patients, including CVD in $9(10 \%)$, non-fatal $\mathrm{MI}$ in $0(0 \%)$ and non-fatal CIE in

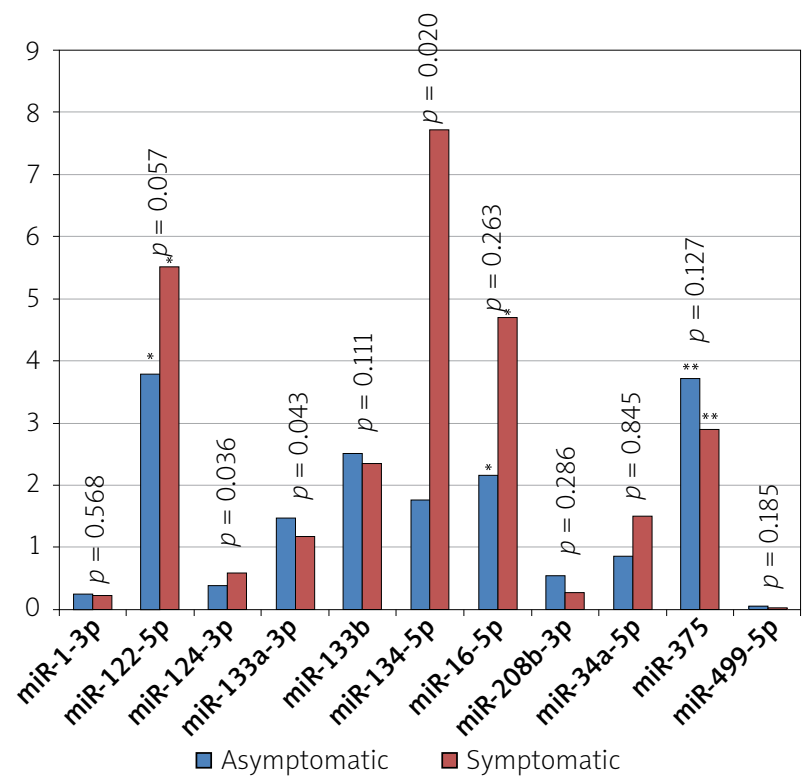

Figure 1. Comparison of miRNA expression in patients with symptomatic vs. asymptomatic course of ICAS

${ }^{*}$ Indicates values with $\times 10^{-2}$, ** values $\times 10^{-1}, "$ values $\times 10^{2}$.

5 (5.6\%). The CVD included: CIE in 3, $\mathrm{MI}$ in 3, and sudden cardiac death in 3 patients.

Patients who suffered from fatal or non-fatal MI had significantly higher levels of miR-1-3p (0.72 \pm 0.64 vs. $0.21 \pm 0.20 ; p=0.048)$, with a non-significant difference for miR-134-5p (6.43 \pm 7.4 vs. $6.5 \pm 36.7 ; p=0.088)$. Fatal or non-fatal $\mathrm{CIE}$ was associated with lower levels of miR$208 b-3 p(0.34 \pm 0.66$ vs. $0.38 \pm 0.23 ; p=0.012)$. There was a statistically significant lower level of miR-208b-3p

Table II. Comparison of ICAS degree as well as plaque morphology and surface in symptomatic vs. asymptomatic subjects

\begin{tabular}{|c|c|c|c|}
\hline Variable & $\begin{array}{c}\text { Symptomatic ICAS } \\
(n=65)\end{array}$ & $\begin{array}{c}\text { Asymptomatic ICAS } \\
(n=27)\end{array}$ & $P$-value \\
\hline$\%$ stenosis on ultrasonography & $78.8 \pm 12.2$ & $81.0 \pm 11.7$ & $0.406^{\#}$ \\
\hline$\%$ stenosis on angiography & $83.5 \pm 16.2$ & $84.3 \pm 12.6$ & $0.609^{\#}$ \\
\hline \multicolumn{4}{|l|}{ Plaque echogenicity, $n$ (\%): } \\
\hline Echolucent & $21(32.3)$ & 3 (11.1) & \\
\hline (Moderately) echogenic & $28(43.1)$ & $19(70.4)$ & $0.033^{*}$ \\
\hline Heterogenic & $16(24.6)$ & $5(18.5)$ & \\
\hline \multicolumn{4}{|l|}{ Plaque surface, $n(\%)$ : } \\
\hline Ulcerated & $23(35.4)$ & $5(18.5)$ & $0.086^{\star *}$ \\
\hline String stenosis & $26(40)$ & $10(37)$ & $0.491^{\star *}$ \\
\hline Thrombus & $9(13.8)$ & $1(3.7)$ & $0.144^{\star *}$ \\
\hline Dissection & $5(7.7)$ & $0(0)$ & $0.168^{\star *}$ \\
\hline
\end{tabular}

\#Mann-Whitney $U,{ }^{*} \chi^{2}$, ${ }^{* *}$ Fisher exact. 
A

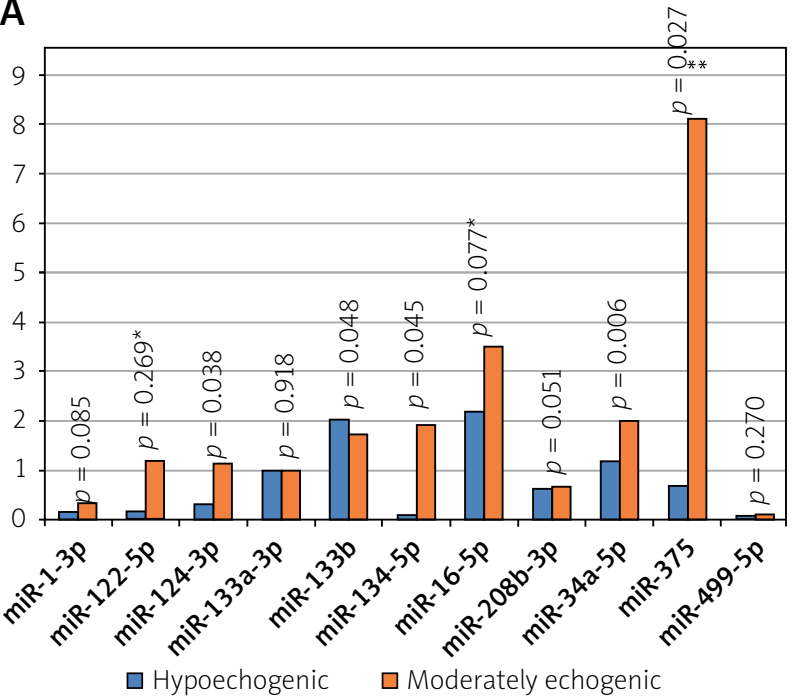

C

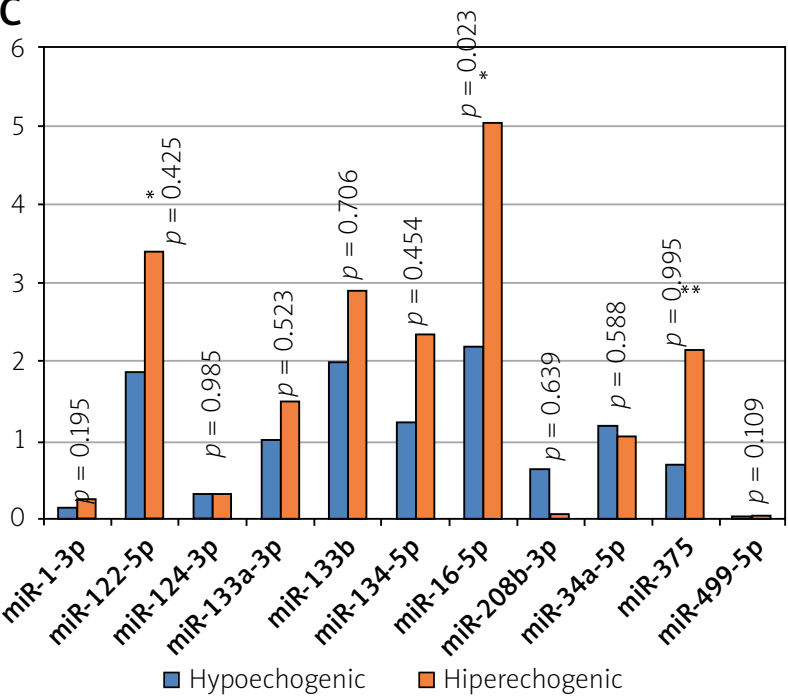

B
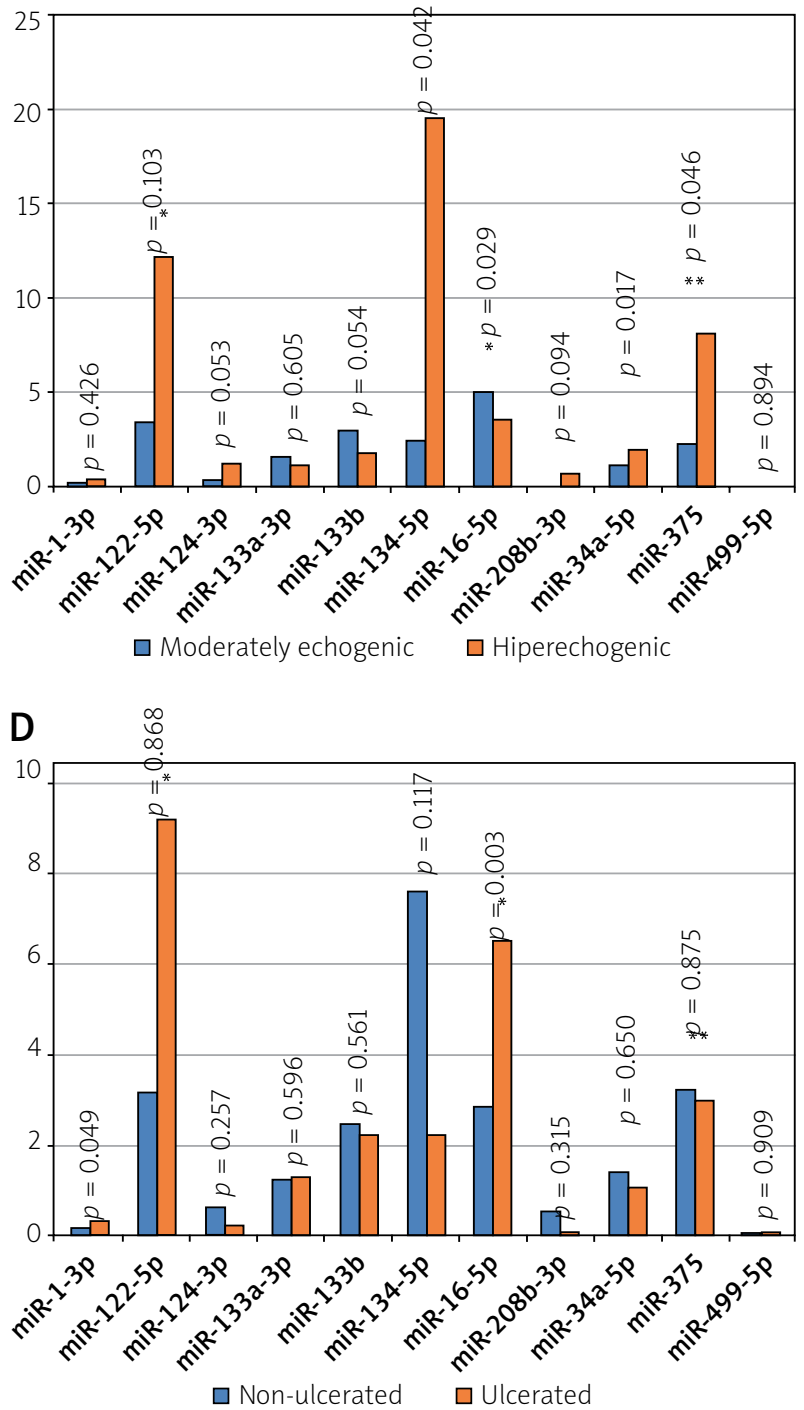

Figure 2. Comparison of miR expression in patients with respect to plaque echogenicity. A - Plaque hypoechogenic vs. moderately echogenic, B - plaque moderately echogenic vs. hyperechogenic, C - plaque hypoechogenic vs. hyperechogenic, $\mathbf{D}-$ ulcerated vs. non-ulcerated plaque *Indicates values with $\times 10^{-2}$, **values $\times 10^{-1}$.

(0.21 \pm 0.51 vs. $0.40 \pm 2.36, p=0.002)$ in subjects with $\mathrm{CVD} / \mathrm{MI} / \mathrm{CIE}$, as compared to the others.

The multivariate Cox proportional hazard analysis revealed fold change of miR-1-3p as a predictor of CVD/MI/ $\mathrm{CIE}(\mathrm{HR}=4.6,95 \% \mathrm{Cl}: 1.61-13.1 ; p=0.004)$, as well as a predictor of CVD or $\mathrm{MI}(\mathrm{HR}=4.84,95 \% \mathrm{Cl}: 1.62-14.5$; $p=0.005$ and $\mathrm{HR}=7.8,95 \% \mathrm{Cl}: 2.01-30.0 ; p=0.003$, respectively) (Table III).

Moreover, ROC analysis showed that miR-1-3p with a cut-off value of 0.508 has only mild discriminating power with respect to prediction of CVD (AUC $=0.634$, 95\% Cl: 0.369-0.900, sensitivity: $55.6 \%$, specificity: 95.1\%) and $\mathrm{MI}(\mathrm{AUC}=0.743,95 \% \mathrm{Cl}: 0.467-1.000$ sensitivity: $66.7 \%$, specificity: $94 \%$ ) (Figure 3 ). Fold changes in levels of miR-133a and miR-133b were independently associated with the risk of future CIE (Table III). However, ROC analysis revealed poor clinical application of these measures, with low sensitivity and specificity and suboptimal AUC (Figure 3; Table IV).

\section{Discussion}

In this prospective study, we measured the levels of circulating miRNAs and found higher levels of brain miRNAs (miR-124-3p and miR-134-5p), while an inverse relationship with miR-133a-3p was found in patients with symptomatic vs. asymptomatic ICAS. There was no correlation between miRNA levels and the degree of ICAS either on ultrasonography or on angiography. We 
Table III. Predictors of CVD/MI/CIE identified by the multivariate Cox proportional hazard analysis

\begin{tabular}{|c|c|c|c|c|}
\hline Cardiovascular event & Prognostic marker & $H R$ & $95 \% \mathrm{Cl}$ & $P$-value \\
\hline Cardiovascular death & miR-1-3p & 4.84 & $1.62-14.5$ & 0.005 \\
\hline Myocardial infarction & miR-1-3p & 7.8 & $2.01-30.0$ & 0.003 \\
\hline Ischemic stroke & $\begin{array}{c}\text { miR-133a-3p } \\
\text { miR-133b }\end{array}$ & $\begin{array}{c}0.3 \\
2.25 \\
\end{array}$ & $\begin{array}{l}0.08-1.12 \\
1.01-5.02 \\
\end{array}$ & $\begin{array}{l}0.074 \\
0.047\end{array}$ \\
\hline Cardiovascular events (CVD/MI/IS) & $\begin{array}{c}\text { miR-1-3p } \\
\text { miR-133a-3p } \\
\text { miR-133b }\end{array}$ & $\begin{array}{l}4.6 \\
0.35 \\
1.99\end{array}$ & $\begin{array}{c}1.61-13.1 \\
0.1-1.22 \\
0.94-4.17\end{array}$ & $\begin{array}{c}0.004 \\
0.101 \\
0.07\end{array}$ \\
\hline
\end{tabular}

A
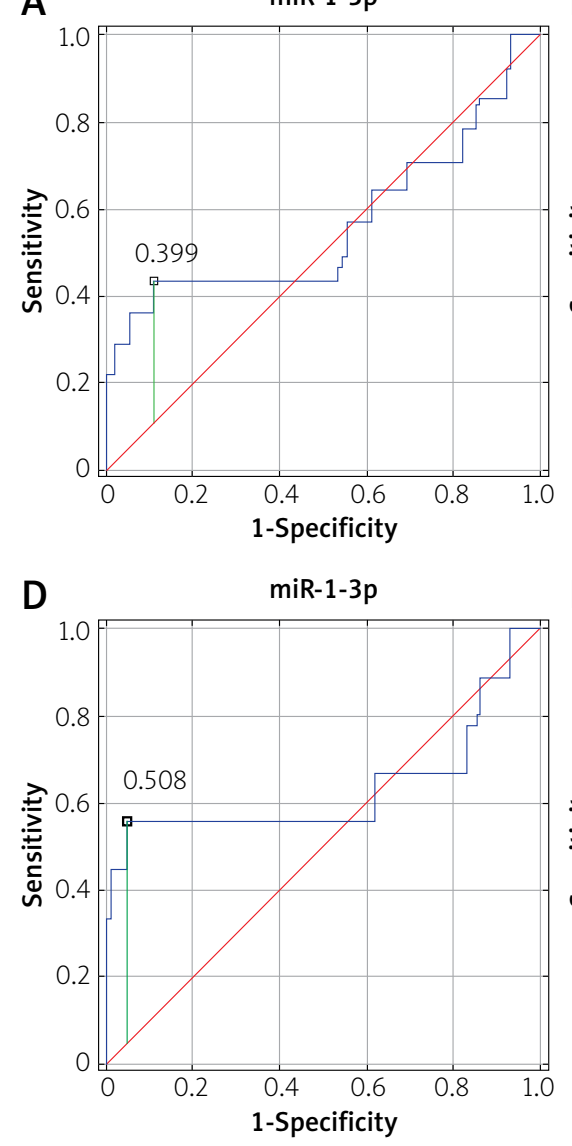

G

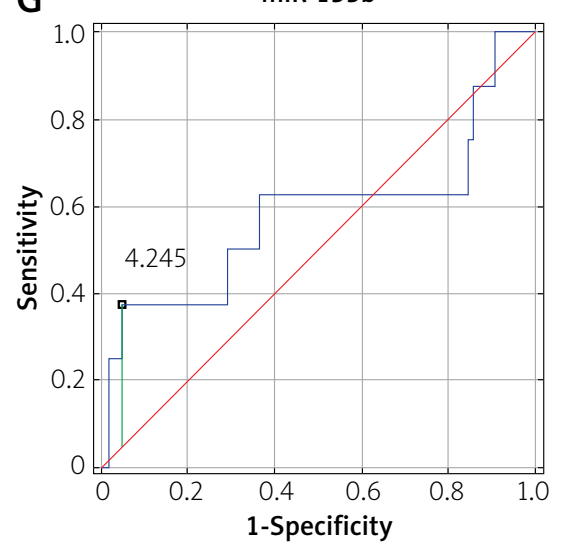

B

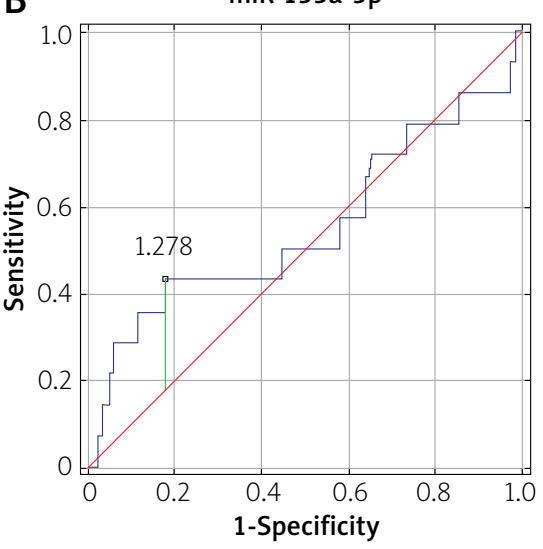

E

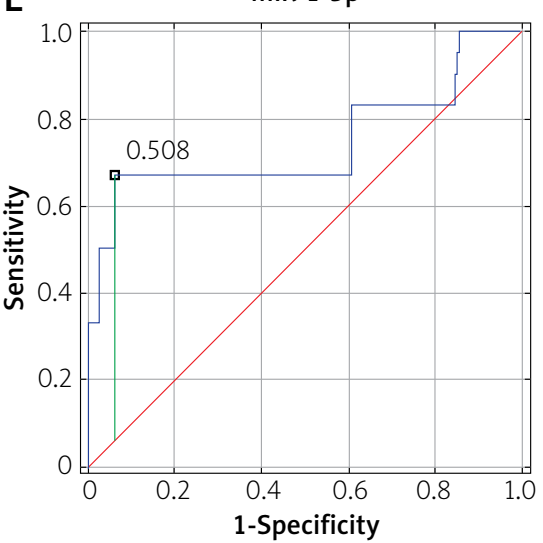

C

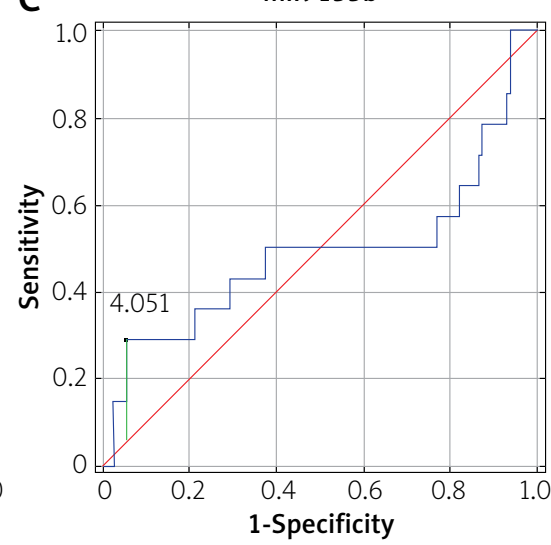

F

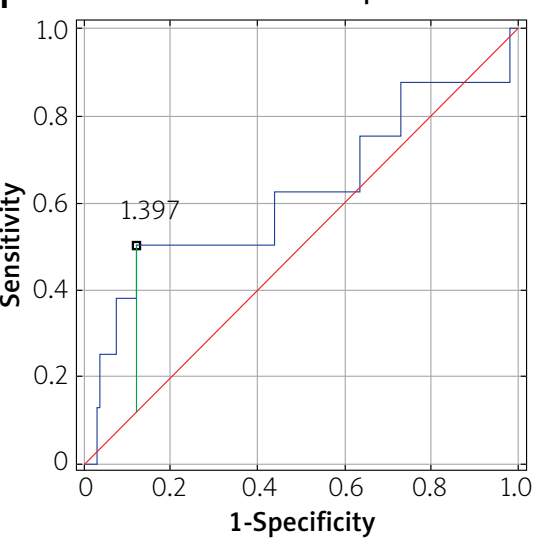

Figure 3. ROC curves for fold change (RQ) in miRNA levels potentially associated with prognostic risk of CVD/MI/CIE (A, B, C), CVD (D), $\mathrm{MI}(\mathrm{E})$, IS $(\mathrm{F}, \mathrm{G})$ 
Table IV. ROC analysis for potential prognostic miRs of future cardiovascular events

\begin{tabular}{|c|c|c|c|c|c|c|}
\hline MACCE & $\begin{array}{c}\text { Potential prognostic } \\
\text { miRNA }\end{array}$ & Cut-off value & AUC & $95 \% \mathrm{Cl}$ & Sensitivity (\%) & Specificity (\%) \\
\hline Cardiovascular death & miR-1-3p & 0.508 & 0.634 & $0.369-0.900$ & 55.6 & 95.1 \\
\hline Myocardial infarction & miR-1-3p & 0.508 & 0.743 & $0.467-1.000$ & 66.7 & 94 \\
\hline Ischemic stroke & $\begin{array}{l}\text { miR-133a-3p } \\
\text { miR-133b }\end{array}$ & $\begin{array}{l}1.371 \\
2.087\end{array}$ & $\begin{array}{l}0.619 \\
0.581\end{array}$ & $\begin{array}{l}0.373-0.865 \\
0.323-0.839 \\
\end{array}$ & $\begin{array}{c}50.0 \\
62\end{array}$ & $\begin{array}{l}87.8 \\
63.4 \\
\end{array}$ \\
\hline $\begin{array}{l}\text { Cardiovascular event } \\
\text { (CVD/MI/IS) }\end{array}$ & $\begin{array}{c}\text { miR-1-3p } \\
\text { miR-133a-3p } \\
\text { miR-133b }\end{array}$ & $\begin{array}{c}0.399 \\
0.926 \\
209\end{array}$ & $\begin{array}{l}0.560 \\
0.546 \\
0.429\end{array}$ & $\begin{array}{l}0.358-0.762 \\
0.354-0.738 \\
0.289-0.695\end{array}$ & $\begin{array}{c}42.9 \\
50 \\
50\end{array}$ & $\begin{array}{l}89.5 \\
55.3 \\
63.2\end{array}$ \\
\hline
\end{tabular}

identified a number of differences in miRNA levels with respect to plaque morphology. Lastly, we found miR-1-3p and miR-133b as potentially useful in predicting future cardiovascular events, such as CVD, MI, CIE.

To our knowledge, this is one of the few studies in humans to address this important clinical issue in patients with ICAS.

According to the Oxford Vascular Study, risk of CIE recurrence related to ICAS is 3.3-fold higher $(95 \% \mathrm{Cl}$ : $1.5-$ 7.0) at 7 days and 2.9-fold higher $(95 \% \mathrm{Cl}: 1.9-4.5)$ at 3 months, as compared with other CIE subtypes [3]. Thus, our symptomatic population (with the median time from CIE of 26 days) fulfills the criteria of the high risk group of recurrent $\mathrm{CIE}$. The model for predicting the risk of ipsilateral CIE in patients with recently symptomatic ICAS from the Stroke Prevention Research Unit at Oxford University includes parameters of plaque surface, e.g. irregularity, ulceration and stenosis degree, as well as time and clinical atherosclerosis risk factors [8].

Identification of the cellular signaling mechanisms between circulating miRNAs and proinflammatory microparticles - transport vehicles for large numbers of specific miRNAs, which have been associated with cardiovascular diseases [30, 31], which are associated with production of the inflammatory, hemorrhagic lesions of mature heterogenic plaques - will help significantly in our understanding of the differences in regions susceptible to rupture and thrombosis. This may help to predict the risk of plaque rupture and guide intervention to patients who will most benefit from intervention on coronary and carotid arteries. As we found previously, in subjects with $\mathrm{MI}$, high levels of brain-derived miRNAs - miR-124-3p (AUC $=0.787, p<0.001)$, miR-133b (AUC $=0.704, p=0.006)$, and miR-134-5p ( $A \cup C=0.686, p=0.016)$ - differentiated an occluded from a patent coronary artery, irrespective of MI type: ST- or non-ST-elevation acute coronary syndrome [29].

The issue of plaque transformation from a stable to an unstable plaque, which is prone to rupture, is a key player in symptom induction. Maitrias et al. found that miR-100, miR-125a, miR-127, miR-133a, miR-145, and miR-221 were significantly overexpressed in symptomatic vs. asymptomatic carotid plaques removed during CEA of 30 patients, which suggested a potential regulatory role for these miRNAs in evolution of the plaque towards growth, instability and rupture [16]. This observation was confirmed by a study of Cipollone et al. who identified upregulation of miR-100, miR-127, miR-145, miR-133a, and miR-133b in symptomatic ICAS removed during CEA [32].

Kim et al., in a group of 120 patients, found, among 5 analyzed miRNAs (miR-17, miR-21, miR-106a, miR-126, and miR-200b), a significantly increased level of miR-17 in acute $\mathrm{CIE}$ patients, while miR-126 levels had a positive correlation with cerebral atherosclerosis $(r=0.254, p=$ 0.021) [15].

In a mouse model of atherosclerotic plaque instability, Chen et al. evidenced that carotid artery unstable plaques are associated with intra-plaque hemorrhage, large necrotic cores, rupture of fibrous caps and luminal thrombosis. Chen et al. demonstrated miR-322 expression as a potential pathogenic factor of plaque instability and also as a therapeutic target in order to prevent plaque rupture [17].

Although exact histopathological composition of the carotid atherosclerotic plaque cannot be judged by echogenicity obtained on ultrasonography, carotid ultrasonography helps to distinguish soft (lipid-rich and thrombotic) from hard (fibrotic and calcified) plaques, indicate irregular and ulcerated plaques, and estimate the degree of ICAS $[11,12]$.

In fact in our study, patients with symptomatic ICAS showed higher prevalence of echolucent and thrombotic plaques, as compared to asymptomatic patients. Consistently, in patients with soft and echolucent vs. moderately echogenic plaques, we observed significantly altered serum levels of miR-124-3p ( $p=0.038)$, miR-134-5p ( $p=0.045), \operatorname{miR}-34 a-5 p(p=0.006), \operatorname{miR}-375$ ( $p=0.027)$ and miR-133b ( $p=0.048)$, as well as non-significant differences for miR-16-5p ( $p=0.077)$, miR-208b-3p ( $p=$ $0.051)$ and miR-1-3p ( $p=0.085)$. In calcified hyperechogenic vs. moderately echogenic plaques changes in levels of miR-34a-5p ( $p=0.017)$, miR-134-5p ( $p=0.042)$, miR$375(p=0.046)$, and miR-16-5p ( $p=0.029)$ were noted. Importantly, ulcerated plaques were characterized by higher expression of miR-1-3p and miR-16-5p.

We believe that carotid plaque instability and rupture may be the local expression of a widespread vascular instability, as also hypothesized by other researchers [33]. 
E.g., nonculprit plaque ruptures were observed in $20 \%$ of patients with coronary artery disease and were associated with pancoronary vulnerability and a higher 1-year revascularization rate [34].

Our present study demonstrates that a so-called vulnerable patient can be potentially predicted by miRNA expression. Ulcerated carotid plaques were associated with higher levels in miR-1-3p, which was an independent predictor associated with risk of future CVD, MI and composite end point (CVD/MI/CIE), whereas miR-133b was associated with incidence of future CIE, albeit with poor discriminating power as calculated by the ROC analysis.

Wang et al. demonstrated that microRNA-1 post-transcriptionally represses the expression of superoxide dismutase (SOD1), Gclc, and G6PD, which is likely to contribute to the increased reactive oxygen species (ROS) level and the susceptibility to oxidative stress of the hearts of miR-1 transgenic mice [35].

The potential prognostic value of circulating miRNAs in cardiovascular diseases was also postulated by other investigators [36, 37]. In a group of 114 stroke patients, Yang et al. revealed miR-107, miR-128b and miR-153 as potentially valuable biomarkers for stroke patients with the AUC values of $0.971,0.881$ and 0.738 , respectively [38]. What is more, the same study showed a correlation between those miRNA levels and NIHSS score. The other study by Long revealed the ability of miR-30a and miR126 to differentiate stroke patients from controls with the AUC of 0.91 and the maximum sensitivity of $94 \%$ and specificity of $84 \%$ with the cutoff value of 1.676 for miR$30 \mathrm{a}$ and AUC 0.92 , sensitivity of $92 \%$ and specificity of $84 \%$ for the threshold value of 1.75 for miR-126 [39].

The study by Kim et al. proved that miR-17 is elevated in stroke patients and associated with recurrence of $\mathrm{CIE}$ in the future, assessed by the ROC analysis. What is more, dividing the patient group into stroke subtypes revealed higher levels of miR-126 and miR-200b in patients with stroke due to atherosclerotic disease, either large artery atherosclerosis or small artery occlusion [15].

MiR-126 level was also correlated with atherosclerosis scores assessed by MR angiography. In the another paper by Wang et al. [36] the levels of miR-106b, miR4306, miR-320e and miR-320d appeared as considerable biomarkers for early stroke detection with the potential to discriminate patients with suspected ischemic stroke.

To conclude, the recent data from animal and human studies indicate that miRNA profiling in stroke may contribute to risk discrimination of $\mathrm{CIE}$ as well as future cardiovascular events, and thus may be helpful in identification of more precise therapeutic targets.

However, looking at the miscellaneous and sometimes conflicting data from the studies regarding numerous and various miRNA levels depending on the clinical course and plaque morphology, it seems that the cause and significance of these differences remain unknown.

It appears reasonable to identify the repetitive miRNA to establish which miRNAs, if any, have real clinical application.

\section{Conclusions}

Symptomatic patients with ICAS have different brain-derived miRNA levels, as compared to patients with asymptomatic ICAS. miRNA levels differ significantly with respect to carotid plaque echogenicity. Future cardiovascular events are correlated with higher levels of miR-1-3p and miR-133b. The degree of ICAS did not correlate with miRNA levels.

\section{Conflict of interest}

The authors declare no conflict of interest.

\section{References}

1. Chin JH, Vora N. Neurology. The global burden of neurologic diseases. Neurology 2014; 83: 349-51.

2. Benjamin EJ, Blaha MJ, Chiuve SE, et al. Heart disease and stroke statistics-2017 update: a Report from the American Heart Association. Circulation 2017; 135: e146-603.

3. Lovett JK, Coull AJ, Rothwell PM. Early risk of recurrence of subtype of ischemic stroke in population-based incidence studies. Neurology 2004; 62: 569-73.

4. Rothwell PM, Slattery J, Warlow CP. Clinical and angiographic predictors of stroke and death from carotid endarterectomy: systematic review. BMJ 1997; 315: 1571-7.

5. Badacz R, Przewłocki T, Karch I, et al. Low prevalence of collateral cerebral circulation in the circle of Willis in patients with severe carotid artery stenosis and recent ischemic stroke. Adv Interv Cardiol 2015; 11: 312-7.

6. Pedro LM, Fernandes e Fernandes J, Pedro MM, et al. Ultrasonographic risk score of carotid plaques. Eur J Vasc Endovasc Surg 2002; 24: 492-8.

7. Marnane M, Prendeville S, McDonnell C, et al. Plaque inflammation and unstable morphology are associated with early stroke recurrence in symptomatic carotid stenosis. Stroke 2014; 45: 801-6.

8. Stroke Prevention Research Unit, Department of Clinical Neurology, Oxford University Model for predicting the risk of ipsilateral IS in patients with recently symptomatic carotid bifurcation stenosis, http://www.stroke.ox.ac.uk/model/form1.html

9. Biasi GM, Froio A, Deleo G, et al. What have we learned from the Imaging in Carotid Angioplasty and Risk of Stroke (ICAROS) study? Vascular 2004; 12: 62-8.

10. Mathiesen EB, Bonaa KH, Joakimsen O. Echolucent plaques are associated with high risk of ischemic cerebrovascular events in carotid stenosis: the TOMSO study. Circulation 2001; 103: 2171-5.

11. Snow M, Ben-Sassi A, Winter RK, et al. Can carotid ultrasound predict plaque histopathology? J Cardiovasc Surg (Torino) 2007; 48: 299-303.

12. Arai D, Yamaguchi S, Murakami M, et al. Characteristics of carotid plaque findings on ultrasonography and black blood magnet- 
ic resonance imaging in comparison with pathological findings. Acta Neurochir Suppl 2011; 112: 15-9.

13. Przewłocki T, Kabłak-Ziembicka A, Kozanecki A, et al. Polyvascular extracoronary atherosclerotic disease in patients with coronary artery disease. Kardiol Pol 2009; 67 (8A): 978-84.

14. Wrotniak L, Kablak-Ziembicka A, Karch I, et al. Multiterritory atherosclerosis and carotid intima-media thickness as cardiovascular risk predictors after percutaneus angioplasty of symptomatic subclavian artery stenosis. J Ultrasound Med 2016; 35: 1977-84.

15. Kim JM, Jung KH, Chu K, et al. Atherosclerosis-related circulating microRNAs as a predictor of stroke recurrence. Transl Stroke Res 2015; 6: 191-7.

16. Maitrias P, Metzinger-Le Meuth V, Massy ZA, et al. MicroRNA deregulation in symptomatic carotid plaque. J Vasc Surg 2015; 62: 1245-50.e1.

17. Chen YC, Bui AV, Diesch J, et al. A novel mouse model of atherosclerotic plaque instability for drug testing and mechanistic/ therapeutic discoveries using gene and microRNA expression profiling. Circ Res 2013; 113: 252-65.

18. Mitchell PS, Parkin RK, Kroh EM, et al. Circulating microRNAs as stable blood-based markers for cancer detection. Proc Natl Acad Sci U S A 2008; 105: 10513-8.

19. Klimczak D, Pączek L, Jażdżewski K, Kuch M. MicroRNAs: powerful regulators and potential diagnostic tools in cardiovascular disease. Kardiol Pol 2015; 73: 1-6.

20. D’Alessandra Y, Devanna P, Limana F, et al. Circulating microRNAs are new and sensitive biomarkers of myocardial infarction. Eur Heart J 2010; 31: 2765-73.

21. Andreou I, Sun $\mathrm{X}$, Stone $\mathrm{PH}$, et al. MiRNAs in atherosclerotic plaque initiation, progression, and rupture. Trends Mol Med 2015; 21: 307-18.

22. Carpenter JP, Lexa FJ, Davis J. T. Determination of duplex Doppler ultrasound criteria appropriate to the North American Symptomatic Carotid Endarterectomy Trial. Stroke 1996; 27: 695-9.

23. Ferguson GG, Eliasziw M, Barr HW, et al. The North American Symptomatic Carotid Endarterectomy Trial: surgical results in 1415 patients. Stroke 1999; 30: 1751-8.

24. Brinjikji W, Rabinstein AA, Lanzino G, et al. Ultrasound characteristics of symptomatic carotid plaques: a systematic review and meta-analysis. Cerebrovasc Dis 2015; 40: 165-74.

25. Geroulakos G, Ramaswami G, Nicolaides A, et al. Characterization of symptomatic and asymptomatic carotid plaques using high resolution real-time ultrasonography. Br J Surg 1993; 80: 1274-7.

26. Pieniążek P, Tekieli L, Musiałek P, et al. Carotid artery stenting according to the tailored-CAS algorithm is associated with a low complication rate at 30 days: data from the TARGET-CAS study. Kardiol Pol 2012; 70: 378-86.

27. Bourke BM, Crimmins DS. Early control of the distal internal carotid artery during endarterectomy: achievability and results. J Vasc Surg 2002; 36: 70e4.

28. Tendera M, Aboyans V, Bartelink ML, et al. ESC Guidelines on the diagnosis and treatment of peripheral artery diseases. Document covering atherosclerotic disease of extracranial carotid and vertebral, mesenteric, renal, upper and lower extremity arteries: the Task Force on the Diagnosis and Treatment of Peripheral Artery Diseases of the European Society of Cardiology (ESC). Eur Heart J 2011; 32: 2851-906.

29. Gacoń J, Kabłak-Ziembicka A, Stępień E, et al. Decision-making microRNAs (miR-124, -133a/b, -34a and -134) in patients with occluded target vessel in acute coronary syndrome. Kardiol Pol 2016; 74: 280-8.

30. DiehIP, Fricke A, Sander L, et al. Microparticles: major transport vehicles for distinct microRNAs in circulation (CC). Cardiovasc Res 2012; 93: 633-44.

31. Stepien E, Kablak-Ziembicka A, Czyz J, et al. Microparticles, not only markers but also a therapeutic target in the early stage of diabetic retinopathy and vascular aging. Expert OpinTher Targets 2012; 16: 677-88.

32. Cipollone F, Felicioni L, Sarzani R, et al. A unique microRNA signature associated with plaque instability in humans. Stroke 2011; 42: 2556-63.

33. Soeki T, Yamaguchi K, Niki T, et al. Plasma microRNA-100 is associated with coronary plaque vulnerability. Circ J 2015; 79: 413-8.

34. Vergallo R, Uemura S, Soeda $\mathrm{T}$, et al. Prevalence and predictors of multiple coronary plaque ruptures: in vivo 3-vessel optical coherence tomography imaging study. Arterioscler Thromb Vasc Biol 2016; 36: 2229-38.

35. Wang L, Yuan Y, Li J, et al. MicroRNA-1 aggravates cardiac oxidative stress by post-transcriptional modification of the antioxidant network. Cell Stress Chaperones 2015; 20: 411-20.

36. Cortez-Dias N, Costa MC, Carrilho-Ferreira $P$, et al. Circulating miR-122-5p/miR-133b ratio is a specific early prognostic biomarker in acute myocardial infarction. Circ J 2016; 80: 2183-91.

37. Wang Q, Ma J, Jiang Z, et al. Identification of microRNAs as diagnostic biomarkers for acute myocardial infarction in Asian populations: a systematic review and meta-analysis. Medicine (Baltimore) 2017; 96: e7173.

38. Yang ZB, Li TB, Zhang Z, et al. The diagnostic value of circulating brain-specific microRNAs for ischemic stroke. Intern Med 2016; 55: $1279-86$.

39. Long G, Wang F, Li H, et al. Circulating miR-30a, miR-126 and let-7b as biomarker for ischemic stroke in humans. BMC Neurol 2013; 13: 178 . 